\title{
Sonic hedgehog-mediated epithelial-mesenchymal transition in renal tubulointerstitial fibrosis
}

\author{
YONGHENG BAI ${ }^{1}$, HONG LU $^{2}$, CHENGCHENG LIN ${ }^{1}$, YAYA XU ${ }^{1}$, DANNÜ HU ${ }^{1}$, \\ YONG LIANG $^{1}$, WEILONG HONG ${ }^{1}$ and BICHENG CHEN ${ }^{1}$ \\ ${ }^{1}$ Wenzhou Key Laboratory of Surgery and ${ }^{2}$ Department of Laboratory Medicine, \\ The First Affiliated Hospital of Wenzhou Medical University, Wenzhou, Zhejiang 325000, P.R. China
}

Received October 13, 2015; Accepted March 28, 2016

DOI: $10.3892 / \mathrm{ijmm} .2016 .2546$

\begin{abstract}
The sonic hedgehog (SHH) signaling pathway plays a critical role in embryonic development, tissue regeneration and organogenesis. The activation of $\mathrm{SHH}$ signaling produces profibrogenic effects in various tissues, such as the liver and the biliary ducts. However, the role of SHH signaling in renal fibrogenesis remains to be elucidated. For this purpose, in the present study, we evaluated the hypothesis that activated $\mathrm{SHH}$ signaling promotes the acquisition of a myofibroblastic phenotype through the epithelial-mesenchymal transition (EMT), resulting in renal interstitial fibrosis (RIF). Kidney samples from rats subjected to unilateral or bilateral ureteral obstruction exhibited the enhanced expression of SHH-pathway proteins, mesenchymal markers and the decreased expression of epithelial markers. Overactive SHH signaling as well as tubular EMT and RIF in the obstructed kidneys were inhibited by recanalization of the ureter. In vitro, $\mathrm{SHH}$ signaling was activated during EMT induction and extracellular matrix (ECM) deposition was observed in transforming growth factor- $\beta 1$ (TGF- $\beta 1$ )-treated renal tubular epithelial cells [RTECs; NRK-52E cell line]. Exogenous SHH activated $\mathrm{SHH}$ signaling and resulted in the upregulated expression of mesenchymal genes, the profibrogenic cytokine TGF- $\beta 1$, and the downregulated expression of epithelial markers. The blockade of SHH signaling with cyclopamine abolished SHH-mediated EMT as well as the acquisition of a myofibroblastic phenotype, and decreased TGF- $\beta 1$ expression and ECM production. Thus, taken together, these findings demonstrate that the activation of the SHH signaling pathway promotes the induction of EMT and renal tubulointerstitial fibrosis. The pharmacological inhibition of SHH signaling may potentially be of therapeutic value in the management of fibrotic kidney diseases.
\end{abstract}

Correspondence to: Dr Bicheng Chen, Wenzhou Key Laboratory of Surgery, The First Affiliated Hospital of Wenzhou Medical University, Wenzhou, Zhejiang 325000, P.R. China

E-mail: greatsailor@163.com

Key words: sonic hedgehog signaling, renal interstitial fibrosis, epithelial-mesenchymal transition, transforming growth factor- $\beta 1$

\section{Introduction}

Renal interstitial fibrosis (RIF) is a common pathway through which chronic kidney diseases (CKD) progress to end-stage renal disease $(1,2)$. As a dynamic process, RIF is characterized pathologically by the deposition of extracellular matrix (ECM) components in association with the loss of tubular epithelial cells, the accumulation of fibroblasts and the infiltration of inflammatory cells (3). It has been recognized that $\alpha$-smooth muscle actin ( $\alpha$-SMA)-positive myofibroblasts play a crucial role in the progression of RIF following injury, and are responsible for the synthesis of ECM components such as type I and III collagens (1). Myofibroblasts are terminally differentiated cells that drive the development of renal fibrogenesis. Increasing evidence indicates that a large proportion of renal myofibroblasts may originate from differentiated tubular epithelial cells through the epithelial-mesenchymal transition (EMT) $(4,5)$.

The EMT may be an adaptive response of epithelial cells following chronic injury and plays an integral role in the development of RIF $(4,5)$. During the EMT, tubular cells in the kidneys lose their epithelial phenotypes [reduced levels of epithelial markers such as E-cadherin and zonula occluden-1 (ZO-1)], and acquire new characteristic features of mesenchymal cells [the expression of mesenchymal proteins including $\alpha$-SMA and vimentin]. As a multifunctional cytokine with profibrogenic properties, transforming growth factor- $\beta 1$ (TGF- $\beta 1)$ is regarded as one of the important factors in the induction of EMT (3). It has been previously demonstrated that the suppression of the EMT by bone morphogenetic protein-7 (BMP-7), or Smad7, an antagonist of TGF- $\beta 1$ signaling, markedly attenuates fibrotic changes following injury $(6,7)$. Therefore, halting or reversing TGF- $\beta 1$-mediated EMT and the acquisition of a myofibroblastic phenotype are promising, novel strategies for attenuating the progression of RIF.

Sonic hedgehog (SHH) signaling is a stem cell-related signaling pathway that plays an important role in embryonic development, tissue regeneration and organogenesis (8-10). The aberrant activation of the SHH signaling pathway leads to pathological consequences, including the development of various types of human tumors $(11,12)$. In addition, previous studies have also showed that activated $\mathrm{SHH}$ signaling is involved in tissue fibrogenesis $(13,14)$. In sustained and uncontrolled tissue injury, SHH signaling is activated through the 
binding of the ligand $\mathrm{SHH}$, to its membrane receptor patched 1 protein (PTCH1). The binding of the SHH ligand to PTCH1 relieves the inhibition of a signal transducer smoothened (SMO), which is repressed by PTCH1, and then initiates a signaling cascade. As a result, activated SHH signaling promotes cellular proliferation and inhibits apoptosis through enhancing the expression of proliferation-associated genes such as c-Myc and cyclin D (15). As EMT may involve abnormal proliferation, we hypothesized that overactive $\mathrm{SHH}$ signaling also induces the acquisition of a myofibroblastic phenotype through the EMT and results in RIF, which is in agreement with previous studies of fibrogenesis in other types of tissues $(13,14)$.

To explore the role of the SHH signaling pathway in RIF as well as the underlying molecular mechanisms responsible for triggering the fibrogenic response, we examined the expression of SHH-pathway proteins in rats subjected to either unilateral ureteral obstruction (UUO) or bilateral ureteral obstruction (BUO). In addition, the activity of SHH signaling was also evaluated in rats subjected to BUO followed by recanalization of the ureters (referred to as the RBUO group) was also evaluated. In vitro, recombinant protein TGF- $\beta 1$ was used to examine the association between SHH signaling and EMT as well as ECM deposition in renal tubular epithelial cells (RTECs). Moreover, the activity of SHH signaling was regulated by treatment with exogenous $\mathrm{SHH}$ with or without cyclopamine, an Smo inhibitor, in order to further explore the detailed role of this pathway in the induction of EMT and ECM accumulation.

\section{Materials and methods}

Establishment of the animal model. Forty-eight male SpragueDawley rats that weighed approximately 180 to $200 \mathrm{~g}$ (6 to 8 weeks of age) were purchased from the Experimental Animal Center of Wenzhou Medical University (Wenzhou, China). The rats were housed under controlled conditions [temperature $\left(22-25^{\circ} \mathrm{C}\right)$, humidity $(40-60 \%)$ and light cycle $(12 \mathrm{~h}$ dark/ light)] and were fed standard rat chow and water, except for one day of fasting prior to the operation. The weight-matched rats were randomly assigned to two groups: i) an obstruction group and ii) a recanalization group. These two groups were further divided into sub-groups, each containing 12 rats. The obstruction group was divided into the following sub-groups: sham-operation (for 7 days) and UUO (for 7 days). The recanalization group was divided into the following sub-groups: BUO for 1 day and RBUO (BUO for 1 day and then recanalization of the ureters for 7 days). UUO and BUO surgery were performed as previously described $(16,17)$, and the longitudinal axis of the kidneys was examined. In the sham-operation group, the left ureter was only dissociated rather than ligated. In the rats in the BUO or UUO groups, the ureter was ligated using a vein clamp at approximately upper $1 / 3$ near the kidney. In the rats in the RBUO group, both sides of the ligated ureter were open again. All rats were sacrificed by cervical dislocation and were anesthetized by $0.2 \%$ pentobarbital natrium. The kidney samples were excised on day 2 for the BUO group, and on day 8 for the UUO, sham-operation and RBUO groups (after BUO operation).

The animal study protocols were approved by the Institutional Animal Care and Use Committee of Wenzhou Medical University (Wenzhou, China).
Histopathological examination. The kidney specimens were fixed in formalin, embedded in paraffin and cut into 4- $\mu \mathrm{m}$ sections, and then stained with hematoxylin and eosin (H\&E) and Masson's trichrome (both from Yuanye Biotechnology, Shanghai, China). The slides were examined and images were captured using a DM4000B LED microscope system and a DFC 420C 5M digital microscope camera (both from Leica Microsystems $\mathrm{GmbH}$, Wetzlar, Germany). The tubular diameter of kidney specimens with H\&E staining were examined based on the longest dimension of renal tubule.

Immunohistochemical analysis. Immunohistochemical analysis was performed on 4- $\mu$ m-thick sections of the kidney samples that had been dewaxed in xylene and hydrated in graded ethanol (100, 95, 85, and 75\%) and distilled water. Endogenous peroxidase was blocked by $3 \%$ hydrogen peroxide. Antigen retrieval was performed by heating in $0.1 \%$ sodium citrate buffer ( $\mathrm{pH} 6.0$ ). To examine the SHH signaling pathway, anti-SHH (bs-1544R, 1:800; Biogot Technology, Shanghai, China), anti-PTCH1 (sc-9016, 1:1,000), anti-SMO (sc-13943, 1:1,000) and anti-GLI1 (sc-6153, 1:1,000) (all from Santa Cruz Biotechnology, Santa Cruz, CA, USA) antibodies were used. To examine the EMT, anti- $\alpha$-SMA (sc-32251, 1:1,000; Santa Cruz Biotechnology), antiE-caderin (ab53033, 1:1,000; Abcam, Cambridge, MA, USA), and anti-type III collagen (bs-0549R, 1:800; Biogot Technology) antibodies were used. To examine proliferation, anti-proliferating cell nuclear antigen (PCNA) (sc-9857, 1:1,000; Santa Cruz Biotechnology) antibody was used. Immunohistochemical studies were semiquantitatively or quantitatively assessed by two independent investigators in a blinded manner.

Cell culture and drug treatment. The normal rat kidney tubule epithelial (NRK-52E) cell line was obtained from the Cell Bank of Chinese Academy of Sciences (Shanghai, China). The NRK-52E cells were maintained in Dulbecco's modified Eagle's medium supplemented with $5 \%$ fetal bovine serum (FBS), $100 \mathrm{U} / \mathrm{ml}$ penicillin and $100 \mu \mathrm{g} / \mathrm{ml}$ streptomycin (all from Invitrogen, Carlsbad, CA, USA). The NRK-52E cells were seeded on six-well culture plates to approximately $70 \%$ confluence in the complete medium containing 5\% FBS for $24 \mathrm{~h}$, which was then replaced with serum-free medium for $24 \mathrm{~h}$ prior to treatment with $5 \mathrm{ng} / \mathrm{ml} \mathrm{TGF}-\beta 1$, or $10 \mathrm{ng} / \mathrm{ml} \mathrm{SHH}$ (both from PeproTech Rocky Hill, NJ, USA) with or without $5 \mu \mathrm{mol} / 1$ cyclopamine (Merck Chemicals, Darmstadt, Germany).

Immunofluorescence staining. The NRK-52E cells were cultured with TGF- $\beta 1$ or SHH with or without cyclopamine in the six-well plates containing glass slides and were then washed with PBS and fixed with $4 \%$ paraformaldehyde (Sigma-Aldrich, St. Louis, MO, USA) at $4^{\circ} \mathrm{C}$ for $30 \mathrm{~min}$. Following permeabilization with $0.1 \%$ Triton $\mathrm{X}-100$ for $10 \mathrm{~min}$, the specimens were washed with PBS and then blocked with $10 \%$ FBS in order to eliminate non-specific fluorescence. Immunofluorescence staining was performed using anti-type III collagen (1:800), $\alpha$-SMA $(1: 1,000)$, E-cadherin $(1: 1,000)$, PTCH1 $(1: 1,000)$, SMO $(1: 1,000)$, GLI1 $(1: 1,000)$, and PCNA $(1: 1,000)$ as the primary antibody, and the cell preparations were incubated with DyLight 488/594-labeled secondary antibodies. The immunocytochemical samples were semiquantitatively or quantitatively assessed by two independent investigators in a blinded manner. 
Table I. Two-step RT-qPCR primers.

\begin{tabular}{|c|c|c|c|}
\hline Gene & Sequence $\left(5^{\prime} \rightarrow 3^{\prime}\right)$ & GenBank accession no. & Length (bp) \\
\hline Shh & $\begin{array}{l}\text { F: ACAAGAAACTCCGAACGATT } \\
\text { R: ACAAGAAACTCCGAACGATT }\end{array}$ & NM_017221 & 183 \\
\hline Gli1 & $\begin{array}{l}\mathrm{F} \quad \text { CCTCGTGGCTTTCATCAACTCT } \\
\mathrm{R}: \text { GAAGCATCATTGAACCCTGAGTAGA }\end{array}$ & XM_006241443.2 & 185 \\
\hline Ptch1 & $\begin{array}{l}\text { F: TCCAGCCGACCCAGATTG } \\
\text { R: ACATAGTCGTAGCCCCTGAAGTG }\end{array}$ & NM_053566.1 & 252 \\
\hline Smo & $\begin{array}{l}\text { F: TGTGGCTCAGGTAGATGG } \\
\text { R: GGTGGTTGCTCTTGATGG }\end{array}$ & NM_012807.1 & 170 \\
\hline Coll $\alpha 1$ & $\begin{array}{l}\text { F: GATCCTGCCGATGTCGCTAT } \\
\text { R: GGAGGTCTTGGTGGTTTTGTATTC }\end{array}$ & NM_053304.1 & 276 \\
\hline $\operatorname{Col} 3 \alpha 1$ & $\begin{array}{l}\text { F: AAGGCTGAAGGAAATAGC } \\
\text { R: AATGTCATAGGGTGCGATA }\end{array}$ & NM_032085.1 & 147 \\
\hline TGF- $\beta 1$ & $\begin{array}{l}\text { F: AGGCGGTGCTCGCTTTGT } \\
\text { R: GATTGCGTTGTTGCGGTCC }\end{array}$ & NM_021578.2 & 137 \\
\hline TGF- $\beta 1 R$ & $\begin{array}{l}\text { F: TGATCCATCCGTTGAAGAAA } \\
\text { R: CTAGCTGCTCCATTGGCATA }\end{array}$ & XM_006238030.2 & 144 \\
\hline E-cadherin & $\begin{array}{l}\text { F: GTGCCACCACCAAAGATA } \\
\text { R: GGCTGAGACAACCCTAAT }\end{array}$ & NM_031334.1 & 195 \\
\hline$\alpha-\mathrm{SMA}$ & $\begin{array}{l}\text { F: GGCATCCACGAAACCACCT } \\
\text { R: CCGCCGATCCAGACAGAAT }\end{array}$ & NM_031004.2 & 212 \\
\hline Vimentin & $\begin{array}{l}\text { F: TGACCGCTTCGCCAACTAC } \\
\text { R: CGCAACTCCCTCATCTCCTC }\end{array}$ & NM_031140.1 & 141 \\
\hline$\beta$-actin & $\begin{array}{l}\text { F: CCCATCTATGAGGGTTACGC } \\
\text { R: TTTAATGTCACGCACGATTTC }\end{array}$ & NM_031144.2 & 150 \\
\hline
\end{tabular}

F, forward; R, reverse; Shh, sonic hedgehog; Gli1, GLI family zinc finger 1; Ptch1, patched 1 protein; Smo, smoothened; collagen, type I, $\alpha$ 1, Col1 $\alpha 1$; collagen, type III, $\alpha 1$,Col3 $\alpha 1 ; \alpha$-SMA, $\alpha$-smooth muscle actin.

Reverse transcription-quantitative polymerase chain reaction ( $q R T-P C R)$. Total RNA was extracted from the rat kidneys using TRIzol reagent (Invitrogen), reverse transcribed to cDNA templates using a ReverTra Ace qPCR RT kit (Toyobo Biotechnology, Tokyo, Japan). RT-qPCR was performed using a SYBR-Green Real-Time PCR Master Mix-Plus- (Toyobo Biotechnology). The quality was analyzed on agarose gels, and the quantity was measured using Varioskan Flash (Thermo Fisher Scientific, Waltham, MA, USA). The sequence-specific primers of Shh; GLI family zinc finger 1 (Gli1); Ptch1; Smo; collagen, type I, $\alpha 1$ (Coll $\alpha 1)$; collagen, type III, $\alpha 1$ (Col3 $\alpha 1)$; TGF- $\beta 1$; TGF- $\beta 1 R$; E-cadherin; vimentin and $\alpha$-SMA, listed in Table I, were synthesized by Invitrogen, and $\beta$-actin was used as an endogenous reference gene. The samples were analyzed in triplicate, and the melting curve was examined to verify that a single product was amplified. The quantitative analysis of all samples was performed using the $\Delta \Delta \mathrm{CT}$ value method.

Enzyme-linked immunosorbent assay (ELISA). Suspensions were prepared by the homogenization of the rat kidney tissues and subsequently centrifuged (at $5,000 \mathrm{x} \mathrm{g}$, at $4^{\circ} \mathrm{C}$ for $10 \mathrm{~min}$ ) and the resulting supernatant was collected. Cells treated with $0-50 \mathrm{ng} / \mathrm{ml} \mathrm{SHH}$ were cultured for $24 \mathrm{~h}$, and the culture super- natant fluid was collected. The avidin-biotin complex-ELISA was performed according to the manufacturer's instructions in order to determine the levels of TGF- $\beta 1$ and SHH. The ELISA kits were purchased from XiTang Biotechnology (Shanghai, China). All experiments were repeated at least three times.

Western blot analysis. Whole proteins from the rat kidneys or the cultured cells were collected and western blot analysis was performed using antibodies against PTCH1 (sc-9016, 1:200), SMO (sc-13943, 1:200), $\alpha$-SMA (sc-32251, 1:200), Rac1 (sc-95, 1:200; Santa Cruz Biotechnology) and E-cadherin (1:400). Quantification was performed by measuring the intensity of the signals using Image-Pro Plus software (version 6.0; Media Cybernetics, Silver Spring, MD, USA), and normalized to that of the $\beta$-actin antibody (AP0060, 1:3,000; Biogot Technology).

Statistical analysis. The data are presented as the means \pm SEM. All of the statistical analyses were performed using the Statistical Package for Social Sciences (version 16.0; SPSS Inc., Chicago, IL, USA). To analyze the difference between two groups, the two-sided Student's t-test was used. One-way ANOVA was used when more than two groups were present. A P-value $<0.05$ was considered to indicate a statistically significant difference. 
A

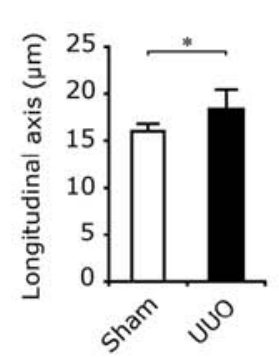

B

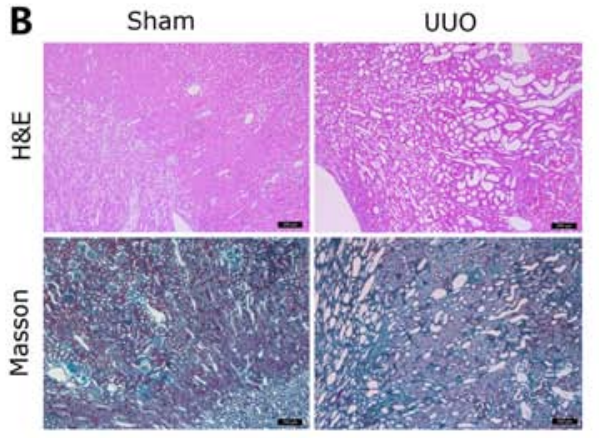

C

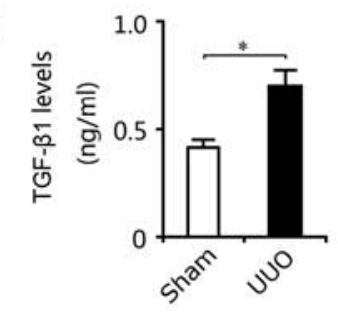

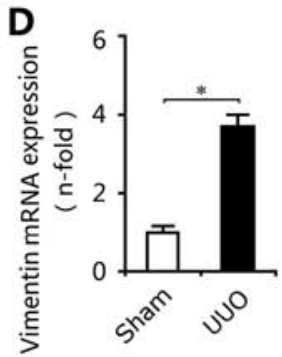
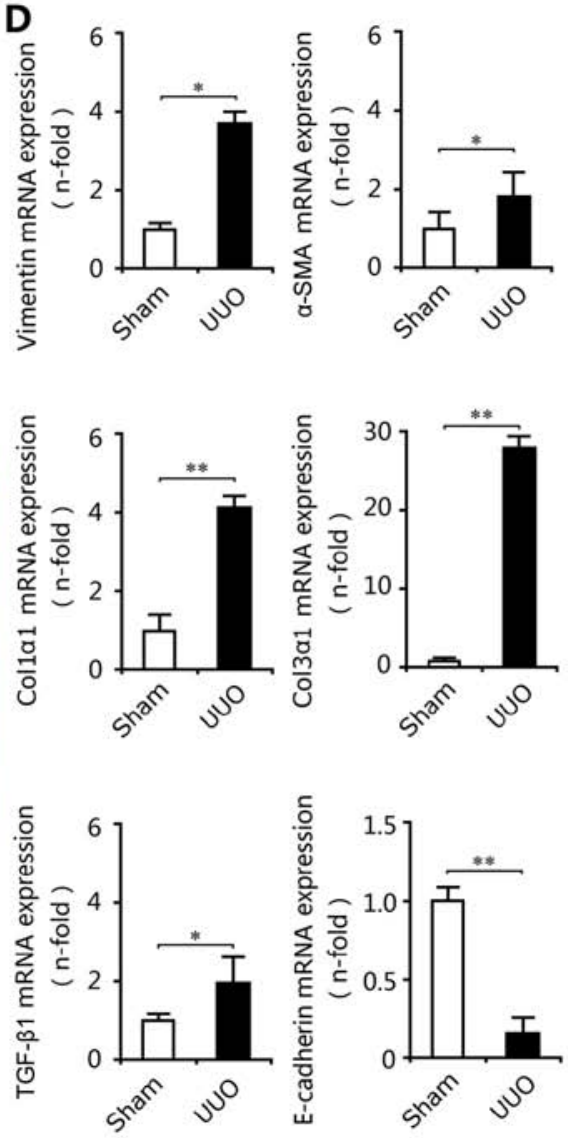

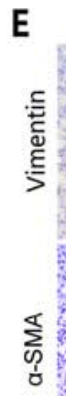

Sham
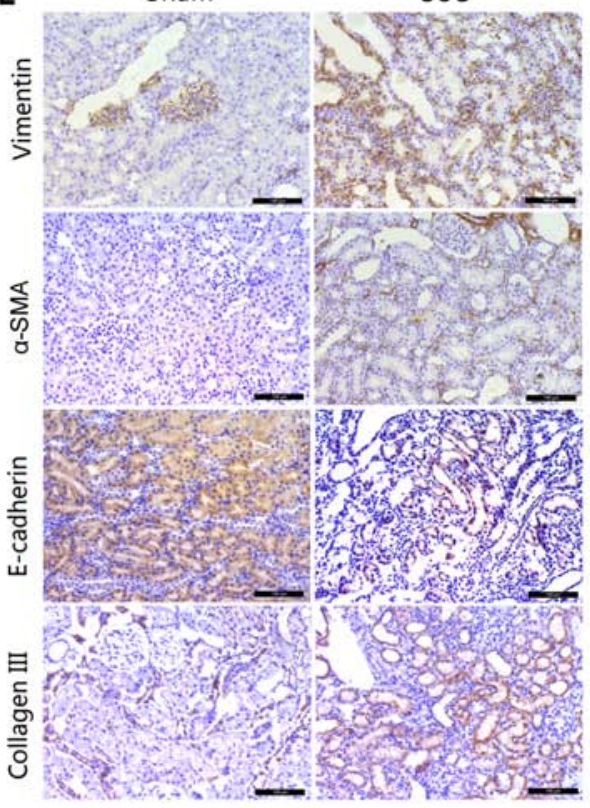

F

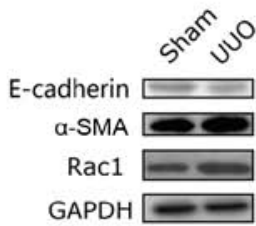

$\square$ E-cadherin/GAPDH a a-SMA/GAPDH

- Rac1/GAPDH

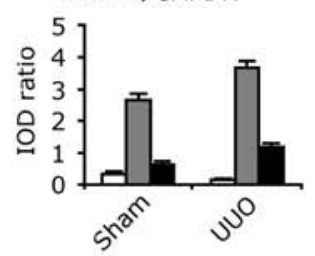

Figure 1. Epithelial-mesenchymal transition (EMT) and renal interstitial fibrosis (RIF) in rats with unilateral ureteral obstruction (UUO). (A) Ureteral obstruction induced marked increases in the longitudinal axis and tubular diameter in the rat kidneys. ${ }^{*} \mathrm{P}<0.05$, ${ }^{* *} \mathrm{P}<0.01 \mathrm{vs}$. the sham group. (B) $\mathrm{H} \& \mathrm{E}$ staining showed obvious kidney injury in the kidneys from the UUO group and Masson's trichrome staining revealed excessive deposition of total collagen. (C) The levels of TGF- $\beta 1$, determined by ELISA, in the rats with UUO were enhanced. Results are expressed as the means \pm SEM ( $=3$ ), ${ }^{*} \mathrm{P}<0.05$ vs. the sham group. (D) RT-qPCR showed that mRNA expression levels of vimentin, $\alpha$-smooth muscle actin ( $\alpha$-SMA), collagen, type I, $\alpha 1$ (Coll $\alpha 1)$, collagen, type III, $\alpha 1$ (Col3 $\alpha 1$ ) and TGF- $\beta 1$ were increased in the UUO group, and the mRNA expression level of E-cadherin was decreased. Results are expressed as the means \pm SEM ( $=6$ ), ${ }^{*} \mathrm{P}<0.05,{ }^{* *} \mathrm{P}<0.01$ vs. the sham group. (E) Upregulated expression of vimentin, $\alpha$-SMA and type III collagen and downregulated expression of E-cadherin in kidney tissues of rats subjected to UUO compared with the sham groups, were determined by immunohistochemical staining. Bar, $100 \mu \mathrm{m}$. (F) Western blot analysis revealed the enhanced protein expression of $\alpha$-SMA and Rac1, and the reduced expression of E-cadherin in the kidneys of rats with UUO.

\section{Results}

SHH signaling activity increases during RIF induced by UUO in rats. As expected, the longitudinal axis and the tubular diameter in the UUO group were significantly increased compared with the sham-operated group (Fig. 1A). In the obstructed kidneys, $\mathrm{H} \& \mathrm{E}$ staining revealed marked tubular dilation and atrophy associated with inflammatory cell infiltration (Fig. 1B, top panels), and Masson trichrome staining revealed severe tubulointerstitial fibrosis (Fig. 1B, lower panels). In addition, the results of ELISA showed that the expression levels of the profibrotic factor TGF- $\beta 1$ in the kidneys from the UUO group were significantly elevated (Fig. 1C). The results of immunohistochemical staining, RT-qPCR and western blot analysis revealed the high mRNA and protein expression of the mesenchymal markers, $\alpha$-SMA, vimentin and Rac1, and the low expression of the epithelial marker E-cadherin in the UUO group (Fig. 1D-F). Moreover, UUO also increased Racl protein expression (Fig. 1F). Racl is regarded as an important indicator of cellular adhesion and invasion (18) and the high expression of Rac1 increases the migration ability of epithelial cells and promotes the transition to myofi- broblasts. Thus, ureteral obstruction induced a myofibroblastic phenotypic change, resulting in excessive ECM deposition and RIF, as demonstrated by the upregulated expression of TGF- $\beta 1$, type I (Coll $\alpha 1)$ and III (Col3 $\alpha 1)$ collagens in the kidney tissues of the rats subjected to UUO (Fig. 1D and E).

In the obstructed kidneys, the enhanced expression of PCNA in the renal cortex, particularly around the renal tubules, revealed that the proliferation of RTECs may involve tubular EMT and RIF (Fig. 2A and B). Thus, we examined the activation of the proliferation-associated SHH signaling pathway in the kidneys from the UUO group. Our results revealed that obstruction not only enhanced the Shh levels (Fig. 2C), but also upregulated the protein expression of SMO, SHH and GLI1, and downregulated the expression of PTCH1 (Fig. 2D). In addition, the mRNA expression levels as indicated by RT-qPCR also confirmed the results of immunohistochemical staining described above (Fig. 2E). Thus, these results showed that $\mathrm{SHH}$ signaling was activated following obstruction. Furthermore, PTCH1 is mainly expressed in the epithelial cells around the renal tubules, which suggests that the activation of SHH signaling may be principally responsible for the induction of the proliferation of RTECs and transdifferentia- 

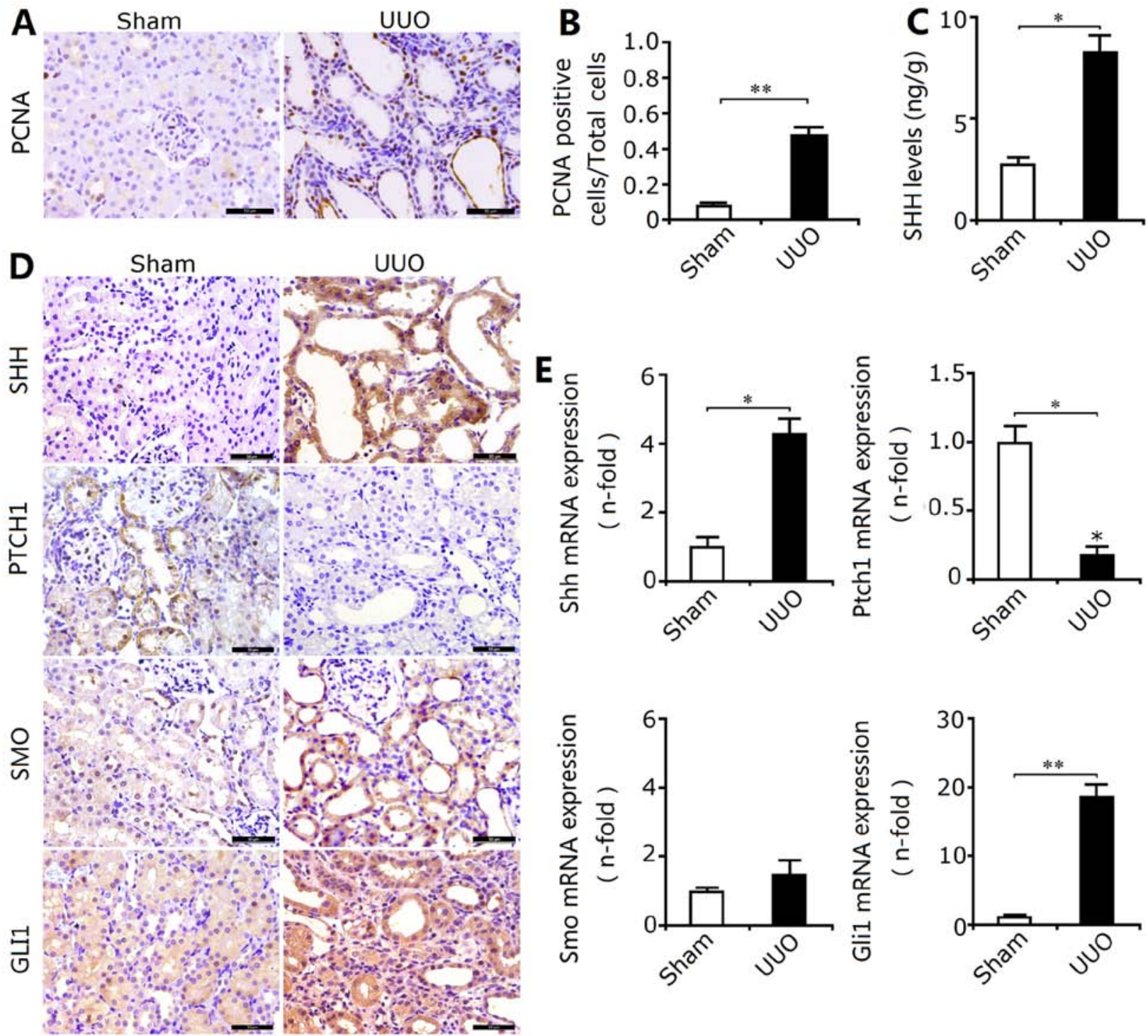

Figure 2. Activation of sonic hedgehog (SHH) signaling during renal interstitial fibrosis (RIF) in rats with unilateral ureteral obstruction (UUO). (A) The expression of proliferating cell nuclear antigen (PCNA) was determined by immunohistochemical staining. Bar, $50 \mu \mathrm{m}$. (B) The ratio of PCNA-positive cells/total cells (determined according to Fig. $2 \mathrm{~A}$ in the rats with UUO) was significantly increased. Results are expressed as the means \pm SEM $(n=10)$, ${ }^{* *} P<0.01$ vs. the sham group. (C) The levels of sonic hedgehog (SHH) determined by ELISA in the rats with UUO were enhanced. Results are expressed as the means \pm SEM ( $\mathrm{n}=3$ ), "P<0.05 vs. the sham group. (D) The location and expression of SHH, patched 1 protein (PTCH1), smoothened (SMO), and GLI family zinc finger 1 (GLI1) were determined by immunohistochemical staining. Bar, $100 \mu \mathrm{m}$. The results showed that the protein expression of SHH, SMO and GLI1 in the rats with UUO was increased, and the expression of Ptch1 was decreased. (E) RT-qPCR revealed that changes in the mRNA expression of Shh, Ptch1, Smo and Gli1 were in accordance with the changes in protein expression. Results are expressed as the means $\pm \operatorname{SEM}(n=6),{ }^{*} \mathrm{P}<0.05,{ }^{* *} \mathrm{P}<0.01$ vs. the sham group.

tion into myofibroblasts. The activation of SHH signaling may be closely associated with the induction of EMT.

Relief of ureteral obstruction reduces $\mathrm{SHH}$ signaling activity, as well as attenuating the EMT and RIF. In this experiment, the rats with BUO were selected as the controls for the rats subjected to recanalization. As observed in the rats subjected to UUO, BUO induced marked tubulointerstitial injury in the kidney tissues. Although the deposition of total collagen determined by Masson's trichrome staining showed that the extent of RIF in the rat kidneys from the BUO group was less severe due to the shorter injury time (Fig. 3A), the upregulated expression of $\alpha$-SMA and type III collagen and the downregulated expression of E-cadherin confirmed the induction of EMT and the excessive deposition of ECM components (Fig. 3B).
Recanalization attenuated the extent of tubulointerstitial injury and reduced the deposition of total collagen. In addition, recanalization also decreased the TGF- $\beta 1$ levels, inhibited EMT induction and reduced ECM accumulation (Fig. 3B-D). Thus, recanalization attenuates obstruction-induced EMT and RIF.

In the rats subjected to UUO, SHH signaling was activated as mentioned above. In the rats subjected to BUO followed by recanalization, we also identified the decreased mRNA expression of Gli1, a target gene of SHH signaling; however, the protein levels of SHH and mRNA expression of Ptch1 did not show any significant differences (Fig. 3E and F). Thus, we speculated that recanalization-induced reduction of renal EMT and RIF may be associated with downregulated $\mathrm{SHH}$ signaling activity in a noncanonical manner. The inhibition of SHH signaling activation may be an important strategy for the attenuation of RIF. 
A
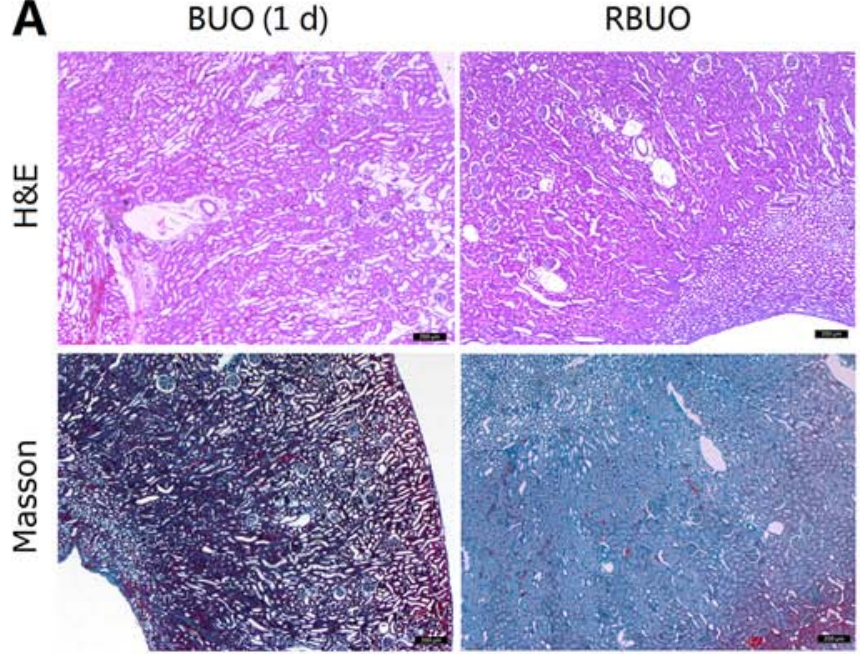

B

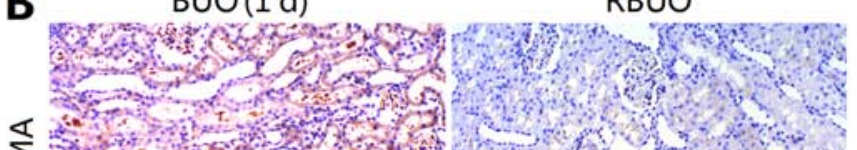

\section{$\sum_{i=1}^{\mathbb{1}}$}

它
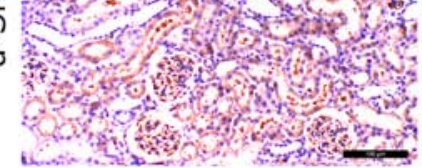

焉
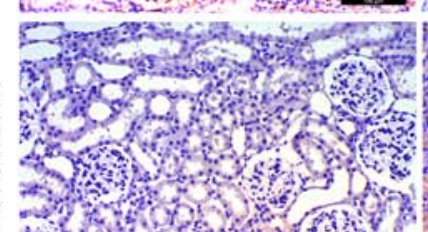

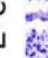

\section{W}

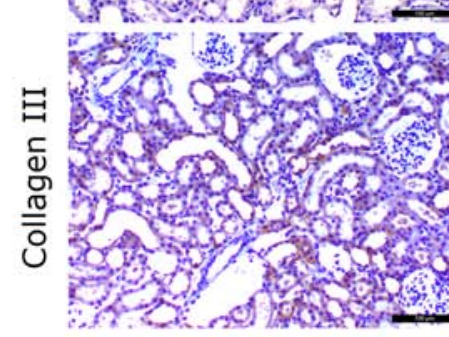

C.

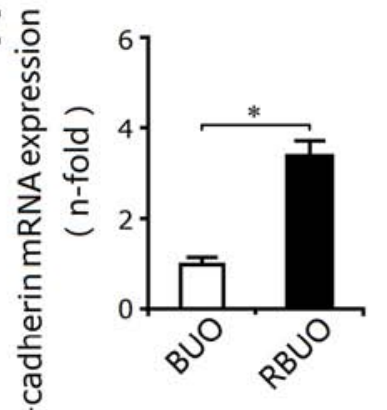

믈
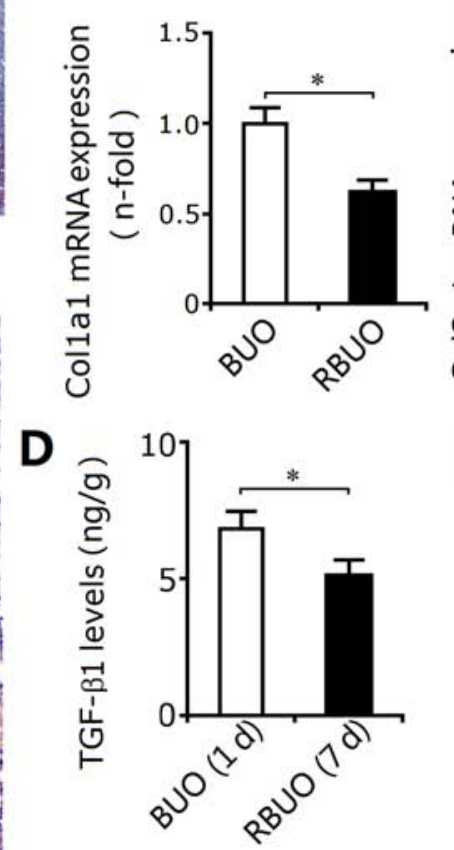

$\mathbf{F}$

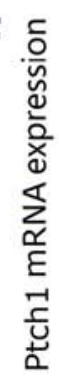
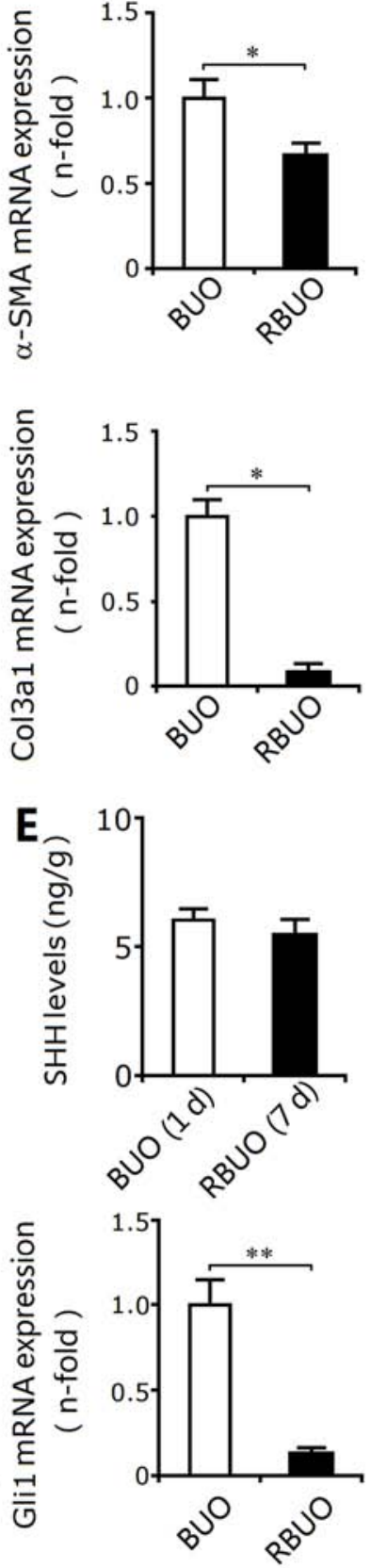

Figure 3. Sonic hedgehog (SHH) signaling activity, epithelial-mesenchymal transition (EMT) and renal interstitial fibrosis (RIF) in the rats subjected to bilateral ureteral obstruction (BUO) followed by recanalization (RBUO group). (A) Compared with the rats subjected to BUO, H\&E and Masson's trichrome staining revealed that recanalization attenuated kidney injury and reduced collagen deposition in the RBUO group, respectively. Bar, $200 \mu \mathrm{m}$. (B) The recanalization operation decreased the protein expression of $\alpha$-smooth muscle actin ( $\alpha$-SMA) and type III collagen (determined by immunohistochemical staining) in the kidneys from the RBUO group, and increased the expression of E-cadherin. Bar, $100 \mu \mathrm{m}$. (C) Recanalization increased the mRNA expression of E-cadherin (determined by RT-qPCR) in the rat kidneys and decreased the expression of $\alpha-S M A, C o l 1 \alpha 1$ and Col3 $\alpha 1$. Results are expressed as the means \pm SEM ( $n=6)$, $P<0.05$ vs. the BUO group. (D) Downregulated levels of transforming growth factor- $\beta 1$ (TGF- $\beta 1$ ) in the RBUO group compared with those in the BUO group. Results are expressed as the means \pm SEM ( $=3),{ }^{*} \mathrm{P}<0.05$ vs. the BUO group. (E) There were no significant differences between the levels of sonic hedgehog (SHH) in the RBUO and BUO groups. (F) Recanalization downregulated the mRNA expression of GLI family zinc finger 1 (Gli1), but not of patched 1 protein (Ptch1). Results are expressed as the means \pm SEM $(n=6),{ }^{* *} \mathrm{P}<0.01$ vs. the BUO group.

Activation of SHH signaling occurs during the EMT and ECM deposition in TGF- $\beta 1$-treated RTECs. In vivo, the above-mentioned findings demonstrated that the activation of the SHH signaling pathway was involved in the induction of EMT and RIF. However, confirmation in vitro is required; thus, we investigated the role of SHH signaling in TGF- $\beta 1$-induced EMT in cultured RTECs (NRK-52E). We found that TGF- $\beta 1$ significantly induced the overexpression of $\alpha$-SMA and type III collagen and reduced the expression of E-cadherin as indicated by immunofluorescence staining (Fig. 4A). Western blot analysis revealed that TGF- $\beta 1$ not only enhanced $\alpha$-SMA expression, but also enhanced Rac1 expression (Fig. 4B). Therefore, the EMT response in the cultured NRK-52E cells was induced by TGF- $\beta 1$.

In addition, TGF- $\beta 1$ also induced the activation of the SHH signaling pathway. As shown in Fig. $4 \mathrm{C}$, the protein expression of 
A

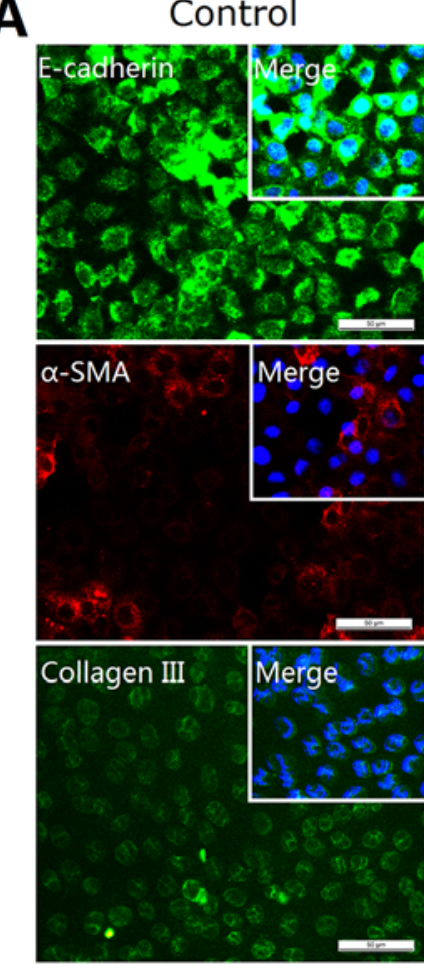

B

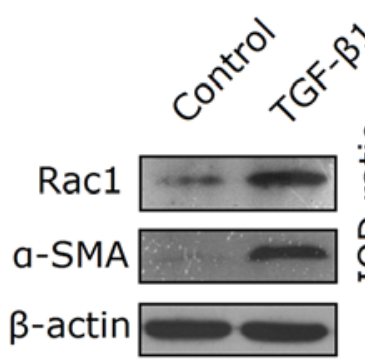

TGF- $\beta 1$

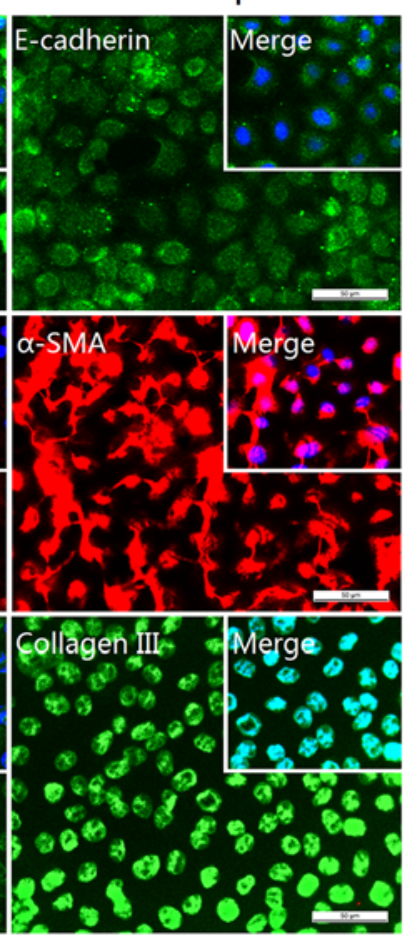

- Rac1/ $\beta$-actin

- $a-S M A / \beta$-actin

1.2

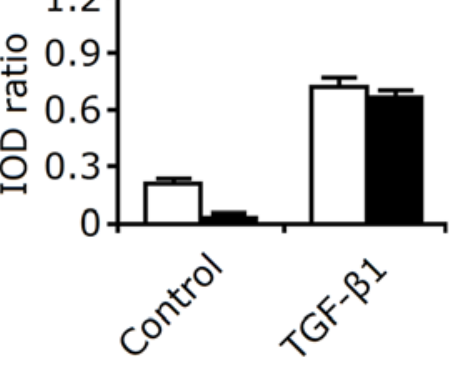

C
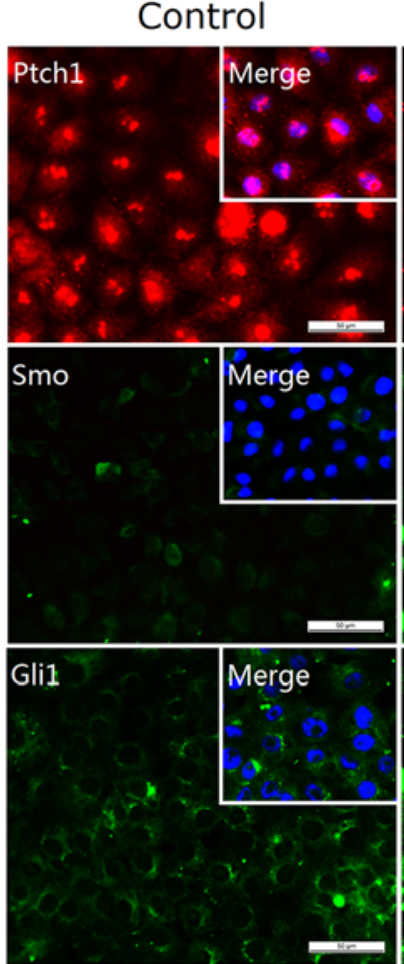

D

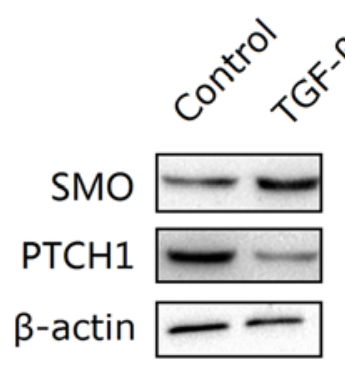

TGF- $\beta 1$

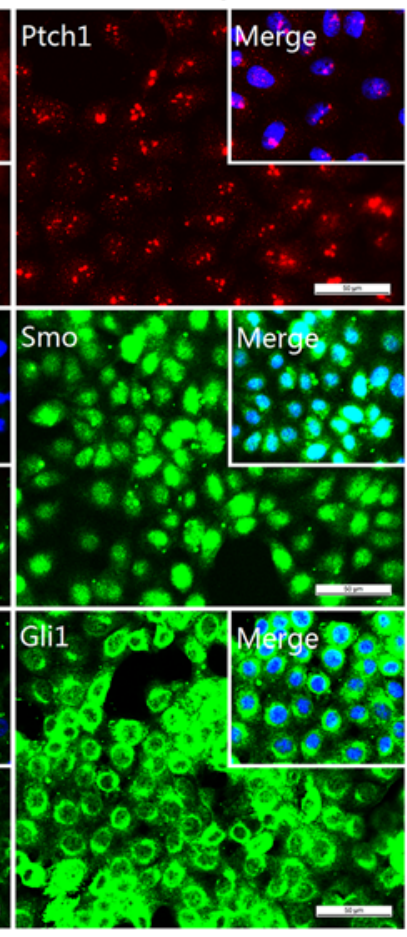

$\square \mathrm{SMO} / \beta$-actin

- PTCH $1 / \beta$-actin

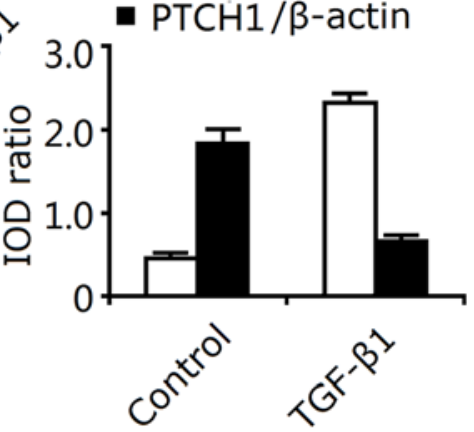

Figure 4. Sonic hedgehog (SHH) signaling activity, epithelial-mesenchymal transition (EMT) and extracellular matrix (ECM) deposition in transforming growth factor- $\beta 1$ (TGF- $\beta 1$ )-treated renal tubular epithelial cells (RTECs). (A) Immunofluorescence staining indicated the upregulated expression of $\alpha$-smooth muscle actin ( $\alpha$-SMA) and type III collagen and downregulated expression of E-cadherin in the TGF- $\beta 1$-treated RTECs. Bar, $100 \mu$ m. (B) Western blot analysis revealed that TGF- $\beta 1$ induced the protein expression of Racl and $\alpha$-SMA. (C) Upregulated expression of smoothened (Smo) and GLI family zinc finger 1 (Gli1) and downregulated expression of patched 1 protein (Ptch1) were indicated by immunofluorescence staining in the TGF- $\beta 1$-treated RTECs. Bar, $100 \mu \mathrm{m}$. (D) Western blot analysis revealed the upregulated protein expression of Smo and downregulated expression of Ptch1 in the TGF- $\beta 1$-treated RTECs.

SMO and GLI1 were significantly increased, and the expression of PTCH1 was decreased in the TGF- $\beta 1$-treated NRK-52E cells. Moreover, western blot analysis revealed the enhanced expression of SMO and the decreased expression of PTCH1 (Fig. 4D). Thus, these findings indicated that the SHH signaling pathway was activated during the EMT induced by TGF- $\beta 1$ treatment.

Exogenous $S H H$ promotes TGF- $\beta 1$ expression and the EMT in RTECs. Given that TGF- $\beta 1$ induced EMT and ECM deposition, and was accompanied by the activation of $\mathrm{SHH}$ signaling, we then aimed to determine whether activated $\mathrm{SHH}$ signaling directly promotes the EMT and ECM deposition. In this experiment using cultured NRK-52E cells, exogenous recombinant protein $\mathrm{SHH}$ was used as an activator of the $\mathrm{SHH}$ pathway. As expected, SHH enhanced the protein expression of SMO and GLI1, and reduced the expression of PTCH1, and thus, activated the SHH signaling pathway (Fig. 5). The activation of SHH signaling resulted in the upregulated expression of PCNA and cellular proliferation (Fig. 6A). This proliferative activity did not induce an increase in cell numbers; however, phenotypic changes were induced. Thus, activated $\mathrm{SHH}$ signaling may play an important role in promoting the deposition of ECM-producing myofibroblasts. Our findings also confirmed this hypothesis. As shown in Fig. 6B, Shh upregulated the protein expression levels of $\alpha$-SMA and type III collagen, and downregulated the expression of E-cadherin. Additionally, Shh also enhanced Rac1 expression (Fig. 6C). Furthermore, this Shh-mediated EMT response may be associated with increased levels of TGF- $\beta 1$ and its receptor TGF- $\beta 1 \mathrm{R}$ (Fig. $6 \mathrm{D}$ and E). Thus, these results suggested that activated $\mathrm{SHH}$ signaling induced the expression of TGF- $\beta 1$ as well as EMT and ECM accumulation. 

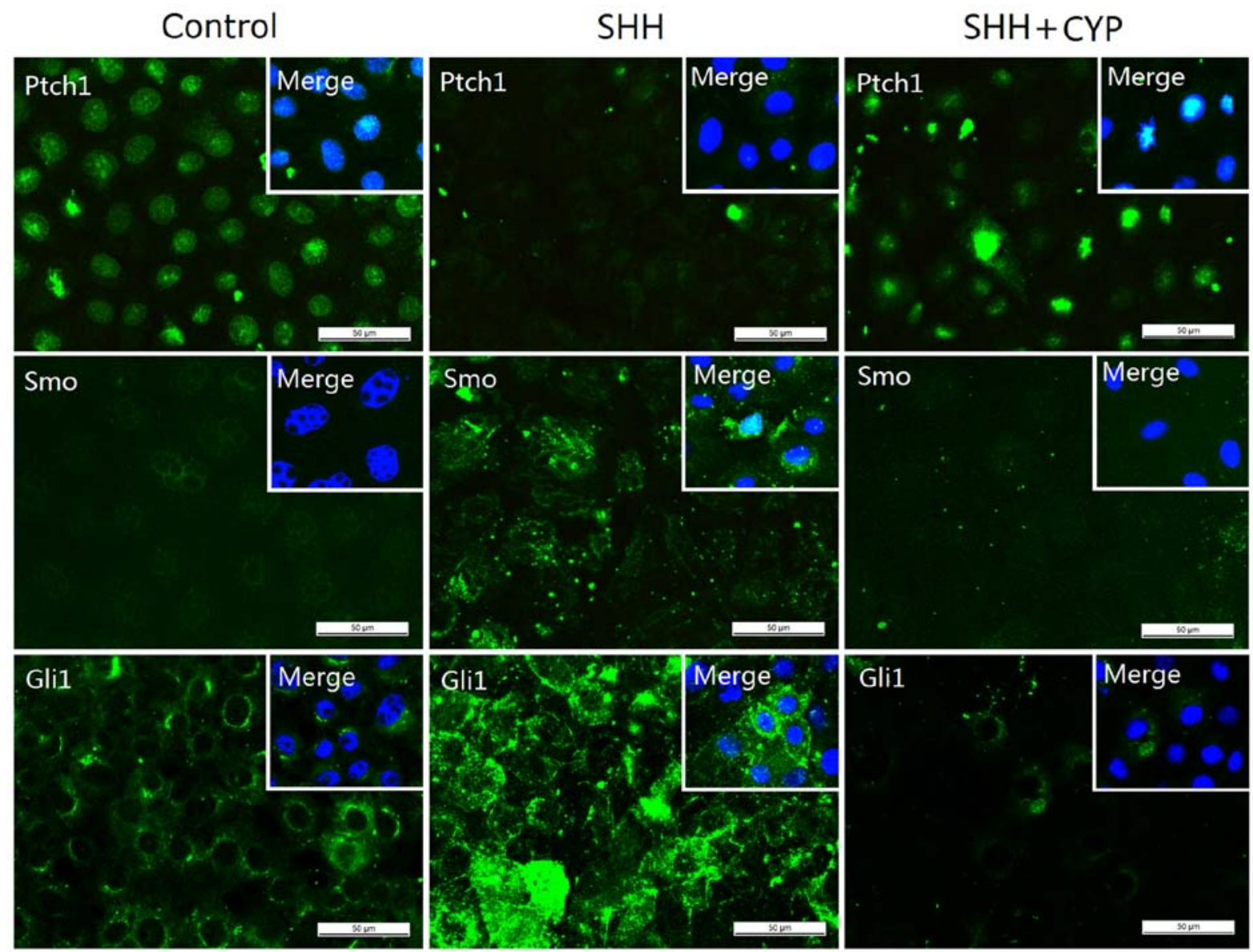

Figure 5. Sonic hedgehog (SHH) signaling activity in sonic hedgehog (SHH) treated-renal tubular epithelial cells (RTECs) in the presence or absence of cyclopamine (Cyp). Immunofluorescence staining indicated the upregulated expression of smoothened (Smo) and GLI family zinc finger 1 (Gli1) and the downregulated expression of patched 1 protein (Ptch1) in the SHH treated-RTECs. However, these changes were inhibited by Cyp treatment. Bar, $50 \mu \mathrm{m}$.

Blockade of SHH signaling inhibits the Shh-mediated EMT and ECM deposition. As stated above, the activated SHH signaling pathway was involved in the induction of EMT and ECM deposition. Herein, we examined whether the downregulated activity of the SHH signaling pathway exerts a protective effect on these fibrotic-like changes. A small molecule antagonist of SHH signaling, cyclopamine was added to the NRK-52E cell culture following SHH treatment. As shown in Fig. 5, cyclopamine significantly inhibited the Shh-mediated downregulation of PTCH1 protein expression as well as the SHH-mediated upregulation of SMO and GLI1 expression. Thus, cyclopamine inhibited the SHH-induced activation of SHH signaling. This inhibition of SHH signaling significantly decreased the proliferation of the NRK-52E cells (Fig. 6A), and resulted in the inhibition of the EMT process and the reduced synthesis of ECM components, as indicated by the downregulated expression of $\alpha$-SMA and type III collagen, and the upregulated expression of E-cadherin (Fig. 6B). Moreover, cyclopamine also reduced the expression levels of TGF- $\beta 1$ and TGF- $\beta 1 \mathrm{R}$ (Fig. $6 \mathrm{E}$ ). Thus, the in vitro blockade of the SHH signaling pathway effectively exerts inhibitory effects on the EMT and ECM deposition.

\section{Discussion}

The present study provides evidence that ureteral obstruction enhances the expression levels of SHH-pathway proteins, mesenchymal markers, and decreases the expression of epithelial markers in the kidney tissues of rats, suggesting that RIF is associated with tubular EMT and the overactivity of the SHH signaling pathway. The EMT as well as fibrotic changes, both of which are associated with SHH signaling, were inhibited by performing recanalization of the ureter. We have also shown that $\mathrm{SHH}$ signaling is activated during the EMT and ECM accumulation in the TGF- $\beta 1$-treated RTECs. Exogenous Shh recombinant protein activated $\mathrm{SHH}$ signaling, resulting in the upregulated expression of mesenchymal genes, the profibrogenic cytokine TGF- $\beta 1$ and the downregulated expression of epithelial markers. Notably, we have found that the blockade of SHH signaling with cyclopamine abolished SHH-mediated EMT, the acquisition of a myofibroblastic phenotype, and decreased TGF- $\beta 1$ expression and ECM production. Thus, the SHH signaling pathway plays an important role in tubular EMT and RIF.

As a key pathway associated with animal development, the SHH signaling pathway has been reported to be involved in the pathogenesis of chronic tissue destruction and impaired renal function $(19,20)$. As SHH signaling plays an important role in the formation of nephrons and kidney development $(21,22)$, some researchers have hypothesized that the aberrant activation of this signaling most likely leads to RIF $(23,24)$. Using a panel of hedgehog-report mice, Fabian et al showed that the ligand Shh is expressed in RTECs, whereas the effector 
A

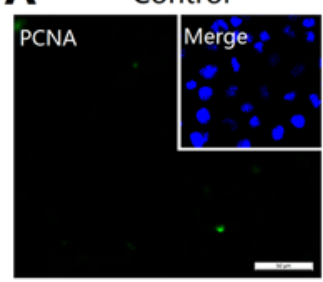

B
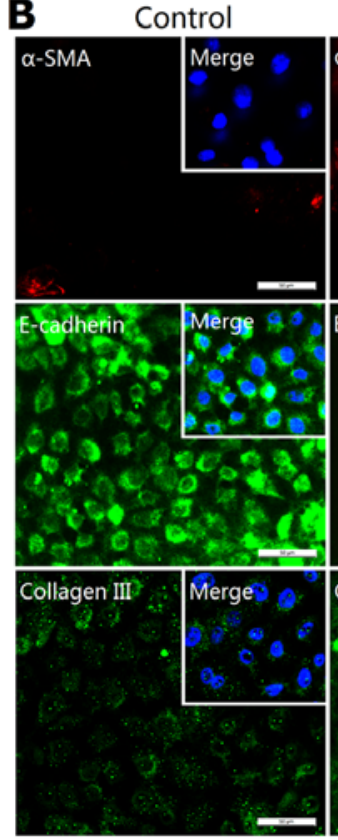

$\mathrm{SHH}$

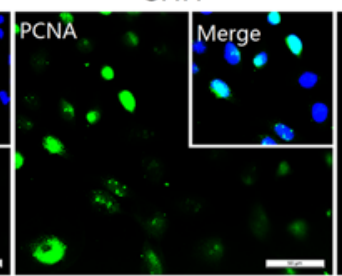

$\mathrm{SHH}$

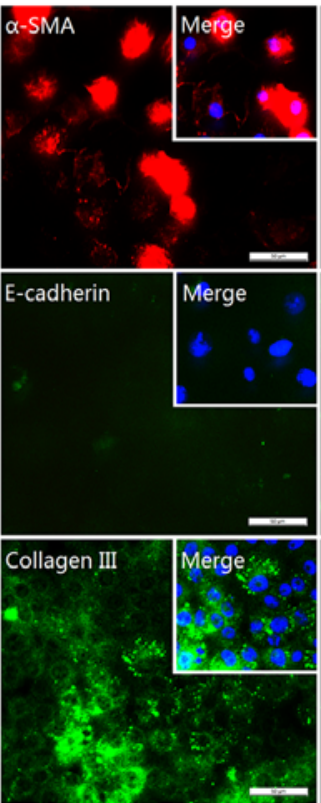

$\mathrm{SHH}+\mathrm{CYP}$

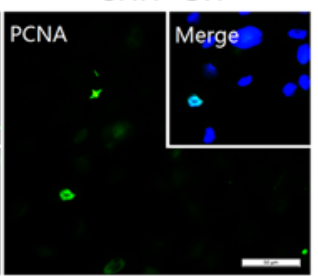

$\mathrm{SHH}+\mathrm{CYP}$

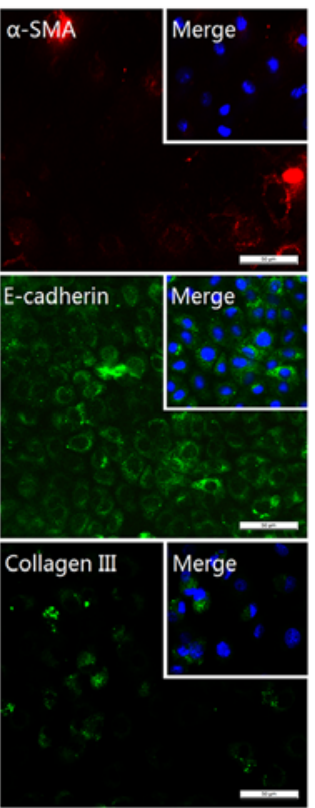

C

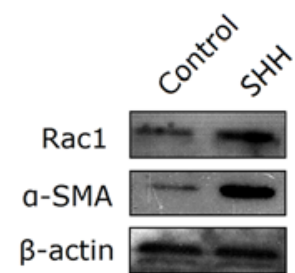

口 Rac1/ß-actin

- a-SMA $/ \beta$-actin

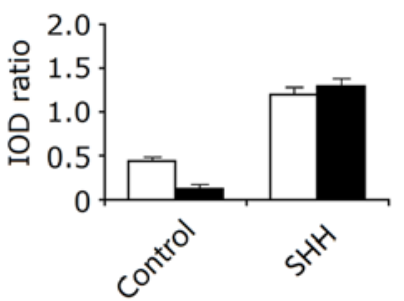

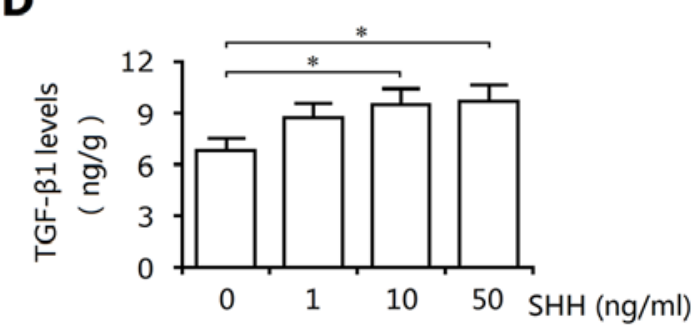

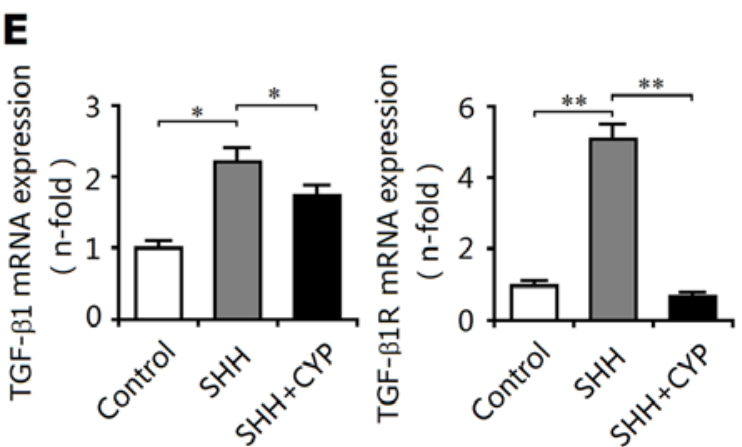

Figure 6. Blockade of sonic hedgehog (SHH) signaling inhibits sonic hedgehog (SHH)-mediated epithelial-mesenchymal transition (EMT) and extracellular matrix (ECM) deposition. (A) Immunofluorescence staining revealed that SHH-induced the upregulated expression of proliferating cell nuclear antigen (PCNA) which was suppressed by cyclopamine (Cyp). (B) SHH increased the protein expression of $\alpha$-smooth muscle actin ( $\alpha$-SMA) and type III collagen and decreased the expression of E-cadherin in renal tubular epithelial cells (RTECs), which was inhibited by Cyp. (C) The expression of Rac1 and $\alpha$-SMA in the RTECs was increased following SHH treatment. (D) SHH enhanced the levels of transforming growth factor- $\beta 1$ (TGF- $\beta 1$ ) in the RTECs (determined by ELISA). Results are expressed as the means \pm SEM ( $n=3),{ }^{*} \mathrm{P}<0.05$, vs. the control group. (E) The mRNA expression of TGF- $\beta 1$ and TGF- $\beta 1 \mathrm{R}$ in the SHH-treated RTECs was increased whereas it was decreased following Cyp treatment. Results are expressed as the means $\pm \mathrm{SEM}(\mathrm{n}=6),{ }^{*} \mathrm{P}<0.05,{ }^{* * *} \mathrm{P}<0.01$ vs. the control group. ${ }^{*} \mathrm{P}<0.05$, ${ }^{* *} \mathrm{P}<0.01$ vs. the SHH-treated group.

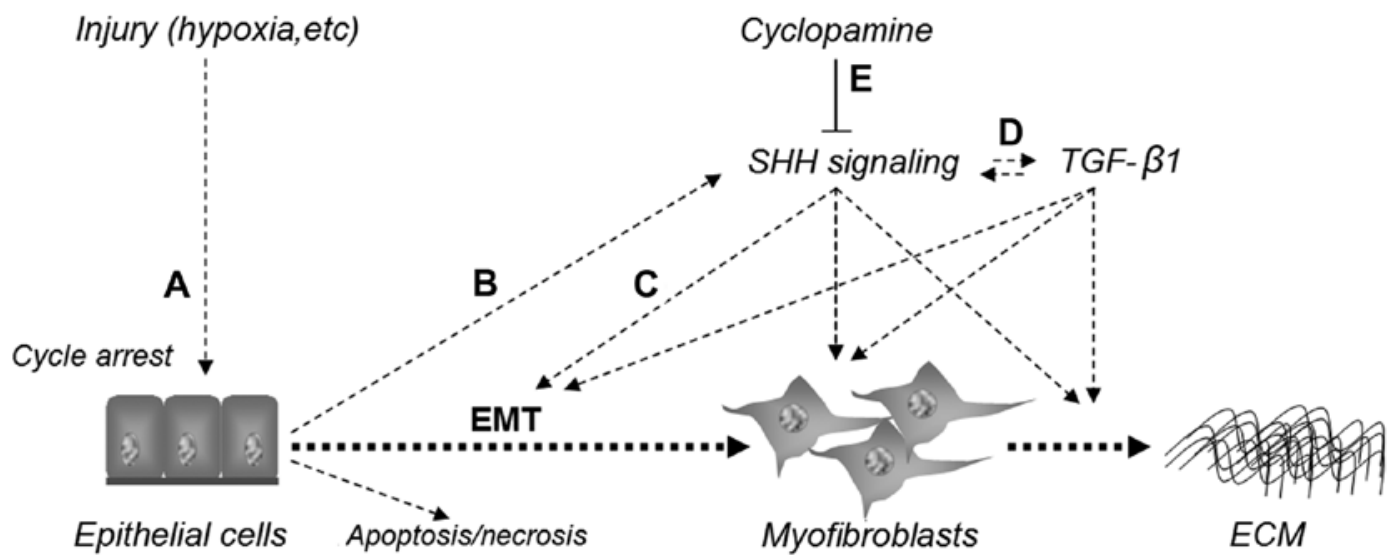

Figure 7. The role of sonic hedgehog (SHH) signaling in epithelial-mesenchymal transition (EMT) induction and extracellular matrix (ECM) deposition. (A) Injuries induce epithelial G2/M cell cycle arrest. (B) Cycle arrest of tubular epithelial cells stimulates the activation of the SHH signaling pathway. (C) The EMT is induced by activated SHH signaling. (D) Activated SHH signaling interacts with the transforming growth factor- $\beta 1$ (TGF- $\beta 1$ ) pathway. (E) Cyclopamine (Cyp) inhibits the activation of SHH signaling, and abolishes the induction of the EMT as well as the acquisition of a myofibroblastic phenotype.

Gli1 is expressed in perivascular fibroblasts and pericytes, suggesting that the SHH signaling pathway is activated in a paracrine manner during renal fibrogenesis (24). Our results supported the finding that the SHH-signaling ligand Shh is mainly expressed in RTECs. However, evidence from immunohistochemical staining in the present study indicated that the 
effector Gli1 is also expressed in tubular epithelial cells as well as in interstitial cells in the kidney tissues of rats subjected to UUO, suggesting that obstruction also induces the activation of renal SHH signaling in an autocrine and a paracrine manner. These results regarding RIF are consistent with a study of liver fibrosis by Yang et al (29), and they identified that the SHH signaling pathway acts in an autocrine manner in the pathogenesis of cirrhosis. In the obstructed kidney, we hypothesized that the GLI1 protein may be a common downstream effector not only of the SHH pathway, but also of other signaling pathways, such as the WNT and BMP pathways (25-27). These activated signaling pathways may regulate Gli1 expression directly or indirectly in tubular epithelial cells, interstitial pericytes and fibroblasts.

Another study, by Ding et al (23), also demonstrated that ureteral obstruction induces SHH expression, predominantly in the renal tubular epithelium of fibrotic kidneys. In addition, they identified interstitial fibroblasts as SHH-responding cells using Gli1 ${ }^{\text {lac }}$ knock-in mice, and in vitro activated SHH signaling promoted myofibroblast activation and matrix production. They hypothesized that SHH signaling-mediated EMT may be the principal cause of myofibroblast accumulation. This deduction was also confirmed by our study; activated SHH signaling is associated with the induction of EMT and the excessive accumulation of ECM components in the kidneys of mice subjected to UUO. Recanalization abolished these above-mentioned changes. In addition, activated SHH signaling, the acquisition of a myofibroblast phenotype and ECM deposition occurred in the TGF- $\beta 1$-treated RTECs. These findings indicated that SHH signaling may be involved in the induction of EMT and RIF.

Although the study of renal fibroblasts by Ding et al showed that activated SHH signaling induces a myofibroblastic phenotype derived from the epithelium (23), further studies from the perspective of epithelial cells are warranted. Thus, in cultured RTECs exogenous SHH recombinant protein wasused to stimulate the activation of SHH signaling. As a result, the expression of PCNA was enhanced. Additionally, the upregulated expression of the mesenchymal marker $\alpha$-SMA, Racl and type III collagen, and the downregulated expression of the epithelial marker E-cadherin were observed in the RTECs. Thus, activated SHH signaling promotes cellular proliferation, and it is accompanied by the transition of RTECs to myofibroblasts. We hypothesized that the proliferation of RTECs, which may be associated with a feedback mechanism, is necessary for the adaptation to injury within the micro-environment and may be one outcome of cell cycle arrest $(28,29)$. However, the abnormal proliferation of RTECs may result in the acquisition of a myofibroblastic phenotype through the EMT $(1,30)$. As an adaptive response of epithelial cells following injury, EMT is increasingly recognized not only as a part of the repair process (unless it is uncontrolled), but also as an integral part of renal fibrogenesis. Compared to epithelial cells, myofibroblasts possess a greater ability to adapt to injury or an abnormal micro-environment. Our in vitro findings confirmed again that activated SHH signaling plays an important role in EMT induction and RIF.

In addition, our results notably showed that TGF- $\beta 1$ may induce the activation of the SHH signaling pathway in RTECs. Similarly, activated SHH signaling may enhance the expression of TGF- $\beta 1$ and its receptor TGF- $\beta 1 \mathrm{R}$, suggesting that there is a feedback loop between the SHH and TGF- $\beta 1$ signaling pathways, and their crosstalk induces EMT and ECM accumulation. However, in order to clarify and confirm this finding, further investigation is also warranted.

Given the importance of SHH signaling in EMT induction and RIF, the blockade of the SHH signaling may exert anti-fibrotic effects. IPI-926, an Smo antagonist, has been reported to possess several advantages including a long half-life, increased potency and oral bioavailability in various animal models (31). However, a study by Fabian et al demonstrated that IPI-926 may completely abolish Gli1 induction whereas it did not affect Gli2 or reduce RIF induced by UUO (24). Thus, IPI-926 may be an ineffective drug for the treatment of RIF. Cyclopamine, another well-characterized Smo inhibitor, is considered to be limited in vivo by its short half-life and off-target effects at higher doses $(32,33)$. However, a study by Ding et al showed that cyclopamine not only prevents fibroblast activation and matrix production in vitro, but also suppresses the expression of Gli1 and matrix genes, and reduces RIF in vivo (23). Thus, cyclopamine efficiently exerts an anti-fibrotic effect. In this study, we found that cyclopamine inhibited the induction of the EMT and reduced the synthesis of ECM components in the cultured RTECs. In addition, cyclopamine also decreased the expression of TGF- $\beta 1$ and its receptor TGF- $\beta 1 R$. These findings suggested that cyclopamine inhibited renal EMT as well as exerting an anti-fibrotic effect in vitro. Unlike its analogue IPI-926; however, the anti-fibrotic effects of cyclopamine and the underlying molecular mechanisms responsible for these effects warrant further study.

Taken together, as shown in Fig. 7, these findings demonstrate that injury induces epithelial $\mathrm{G} 2 / \mathrm{M}$ cell cycle arrest, and then stimulates the activation of the SHH signaling pathway. The activated SHH signaling pathway, which interacts with the TGF- $\beta 1$ pathway, induces the EMT response, promotes the acquisition of a myofibroblastic phenotype and ECM deposition and results in RIF. The blockade of SHH signaling with cyclopamine abolishes Shh-mediated EMT, the acquisition of a myofibroblastic phenotype and decreases TGF- $\beta 1$ expression and ECM production. Thus, SHH signaling may play a critical role in EMT induction and the development of RIF, and the pharmacological inhibition of SHH signaling may play a therapeutic role in the management of fibrotic kidney diseases.

\section{Acknowledgements}

The present study was supported by the Natural Science Foundation of Zhejiang province, China (LQ12H05001 and LY12H05004) and the Wenzhou Municipal Science and Technology Plan Project (Y20110028). The project was also sponsored by the National Natural Science Foundation of China (81572087).

\section{References}

1. Meran S and Steadman R: Fibroblasts and myofibroblasts in renal fibrosis. Int J Exp Pathol 92: 158-167, 2011.

2. Vilayur E and Harris DC: Emerging therapies for chronic kidney disease: what is their role? Nat Rev Nephrol 5: 375-383, 2009.

3. Liu Y: Cellular and molecular mechanisms of renal fibrosis. Nat Rev Nephrol 7: 684-696, 2011.

4. Liu Y: Epithelial to mesenchymal transition in renal fibrogenesis: pathologic significance, molecular mechanism, and therapeutic intervention. J Am Soc Nephrol 15: 1-12, 2004.

5. Liu Y: New insights into epithelial-mesenchymal transition in kidney fibrosis. J Am Soc Nephrol 21: 212-222, 2010. 
6. Saika S, Ikeda K, Yamanaka O, Flanders KC, Ohnishi Y, Nakajima Y, Muragaki Y and Ooshima A: Adenoviral gene transfer of BMP-7, Id2, or Id3 suppresses injury-induced epithelial-to-mesenchymal transition of lens epithelium in mice. Am J Physiol Cell Physiol 290: C282-C289, 2006.

7. Zeisberg M, Hanai J, Sugimoto H, Mammoto T, Charytan D, Strutz F and Kalluri R: BMP-7 counteracts TGF-beta1-induced epithelial-to-mesenchymal transition and reverses chronic renal injury. Nat Med 9: 964-968, 2003.

8. Bhardwaj G, Murdoch B, Wu D, Baker DP, Williams KP, Chadwick K, Ling LE, Karanu FN and Bhatia M: Sonic hedgehog induces the proliferation of primitive human hematopoietic cells via BMP regulation. Nat Immunol 2: 172-180, 2001.

9. Ingham PW and McMahon AP: Hedgehog signaling in animal development: paradigms and principles. Genes Dev 15: 3059-3087, 2001.

10. Pasca di Magliano $M$ and Hebrok M: Hedgehog signalling in cancer formation and maintenance. Nat Rev Cancer 3: 903-911, 2003.

11. Berman DM, Karhadkar SS, Maitra A, Montes De Oca R, Gerstenblith MR, Briggs K, Parker AR, Shimada Y, Eshleman JR, Watkins DN and Beachy PA: Widespread requirement for Hedgehog ligand stimulation in growth of digestive tract tumours. Nature 425: 846-851, 2003.

12. Thayer SP, di Magliano MP, Heiser PW, Nielsen CM, Roberts DJ, Lauwers GY, Qi YP, Gysin S, Fernández-del Castillo C, Yajnik $\mathrm{V}$, et al: Hedgehog is an early and late mediator of pancreatic cancer tumorigenesis. Nature 425: 851-856, 2003.

13. Omenetti A, Porrello A, Jung Y, Yang L, Popov Y, Choi SS, Witek RP, Alpini G, Venter J, Vandongen HM, et al: Hedgehog signaling regulates epithelial-mesenchymal transition during biliary fibrosis in rodents and humans. J Clin Invest 118: 3331-3342, 2008

14. Syn WK, Jung Y, Omenetti A, Abdelmalek M, Guy CD, Yang L, Wang J, Witek RP, Fearing CM, Pereira TA, et al: Hedgehog-mediated epithelial-to-mesenchymal transition and fibrogenic repair in nonalcoholic fatty liver disease. Gastroenterology 137: 1478-1488.e8, 2009.

15. Hooper JE and Scott MP: Communicating with hedgehogs. Nat Rev Mol Cell Biol 6: 306-317, 2005.

16. Bai Y, Lu H, Zhang G, Wu C, Lin C, Liang Y and Chen B: Sedum sarmentosum Bunge extract exerts renal anti-fibrotic effects in vivo and in vitro. Life Sci 105: 22-30, 2014.

17. Pat B, Yang T, Kong C, Watters D, Johnson DW and Gobe G: Activation of ERK in renal fibrosis after unilateral ureteral obstruction: modulation by antioxidants. Kidney Int 67: 931-943, 2005.

18. Katoh H, Hiramoto K and Negishi M: Activation of Racl by RhoG regulates cell migration. J Cell Sci 119: 56-65, 2006.

19. Gill PS and Rosenblum ND: Control of murine kidney development by sonic hedgehog and its GLI effectors. Cell Cycle 5: 1426-1430, 2006.
20. Hu MC, Mo R, Bhella S, Wilson CW, Chuang PT, Hui CC and Rosenblum ND: GLI3-dependent transcriptional repression of Gli1, Gli2 and kidney patterning genes disrupts renal morphogenesis. Development 133: 569-578, 2006.

21. Cain JE and Rosenblum ND: Control of mammalian kidney development by the Hedgehog signaling pathway. Pediatr Nephrol 26: 1365-1371, 2011.

22. Yu J, Carroll TJ and McMahon AP: Sonic hedgehog regulates proliferation and differentiation of mesenchymal cells in the mouse metanephric kidney. Development 129: 5301-5312, 2002.

23. Ding H, Zhou D, Hao S, Zhou L, He W, Nie J, Hou FF and Liu Y: Sonic hedgehog signaling mediates epithelial-mesenchymal communication and promotes renal fibrosis. J Am Soc Nephrol 23: 801-813, 2012.

24. Fabian SL, Penchev RR, St-Jacques B, Rao AN, Sipilä P, West KA, McMahon AP and Humphreys BD: Hedgehog-Gli pathway activation during kidney fibrosis. Am J Pathol 180: 1441-1453, 2012.

25. Borello U, Berarducci B, Murphy P, Bajard L, Buffa V, Piccolo S, Buckingham $\mathrm{M}$ and Cossu G: The Wnt/beta-catenin pathway regulates Gli-mediated Myf5 expression during somitogenesis. Development 133: 3723-3732, 2006.

26. Daoud G, Kempf H, Kumar D, Kozhemyakina E, Holowacz T, Kim DW, Ionescu A and Lassar AB: BMP-mediated induction of GATA4/5/6 blocks somitic responsiveness to $\mathrm{SHH}$. Development 141: 3978-3987, 2014.

27. Nakamura I,Fernandez-Barrena MG, Ortiz-Ruiz MC, Almada LL, $\mathrm{Hu}$ C, Elsawa SF, Mills LD, Romecin PA, Gulaid KH, Moser $\mathrm{CD}$, et al: Activation of the transcription factor GLI1 by WNT signaling underlies the role of SULFATASE 2 as a regulator of tissue regeneration. J Biol Chem 288: 21389-21398, 2013.

28. Wynn TA: Fibrosis under arrest. Nat Med 16: 523-525, 2010.

29. Yang L, Besschetnova TY, Brooks CR, Shah JV and Bonventre JV: Epithelial cell cycle arrest in G2/M mediates kidney fibrosis after injury. Nat Med 16: 535-543, 1p following 143, 2010.

30. Bai Y, Lu H, Hu L, Hong D, Ding L and Chen B: Effect of Sedum sarmentosum Bunge extract on aristolochic acid-induced renal tubular epithelial cell injury. J Pharmacol Sci 124: 445-456, 2014.

31. Tremblay MR, Lescarbeau A, Grogan MJ, Tan E, Lin G, Austad BC, Yu LC, Behnke ML, Nair SJ, Hagel M, et al: Discovery of a potent and orally active hedgehog pathway antagonist (IPI-926). J Med Chem 52: 4400-4418, 2009.

32. Zhang X, Harrington N, Moraes RC, Wu MF, Hilsenbeck SG and Lewis MT: Cyclopamine inhibition of human breast cancer cell growth independent of Smoothened (Smo). Breast Cancer Res Treat 115: 505-521, 2009.

33. Zhao C, Chen A, Jamieson CH, Fereshteh M, Abrahamsson A, Blum J, Kwon HY, Kim J, Chute JP, Rizzieri D, et al: Hedgehog signalling is essential for maintenance of cancer stem cells in myeloid leukaemia. Nature 458: 776-779, 2009. 